\title{
Sunspot Cycle 24 and the Advent of Dalton-Like Minimum
}

\author{
H. S. Ahluwalia and R. C. Ygbuhay \\ Department of Physics and Astronomy, University of New Mexico, MSC07 4220, Albuquerque, NM 87131, USA \\ Correspondence should be addressed to H. S. Ahluwalia, hsa@unm.edu
}

Received 12 October 2011; Revised 2 December 2011; Accepted 15 December 2011

Academic Editor: J. Javaraiah

Copyright $\odot 2012$ H. S. Ahluwalia and R. C. Ygbuhay. This is an open access article distributed under the Creative Commons Attribution License, which permits unrestricted use, distribution, and reproduction in any medium, provided the original work is properly cited.

\begin{abstract}
Ahluwalia and Jackiewicz (2011) have predicted that sunspot cycle 24 will be only half as active as cycle 23, reaching its peak in May $2013 \pm 6$ months. Here, we discuss the timeline for cycle 24 since its onset in December, 2008 and compare it to the timelines for the last ten cycles (14 to 23) of the 20th century; cycle 24 is rising the slowest. We speculate that cycle 24 may herald the onset of a Dalton-like minimum in the 21st century. The implications of this outcome on global temperature change and ensuing socioeconomic and political scenarios are discussed, on the basis of the historical record.
\end{abstract}

\section{Introduction}

The topic of climate change is of great importance and immense interest to the peoples of the world from the socioeconomic and political perspectives. The contribution of the variations in solar activity to the observed climate change is controversial, but past history indicates that at least a part of the observed change may be related to the solar activity and a part of the expected future change may therefore arise from the solar variability as represented by the sunspot numbers (SSNs).

The art of predicting the peak SSNs for a cycle and its rise time has made significant advances since cycle 20 forecasts. Petrovay [1] provides an excellent review of the solar cycle prediction methods and forecasts resulting from them. The empirical methods inspired by the work of Ohl $[2,3]$ made remarkably accurate forecasts for the last two solar cycles. Ahluwalia and Jackiewicz [4] - AJ11 hereafter-describe the unusual decay phase of the sunspot cycle 23 and use a heuristic approach (which is not based on any model) to make a prediction for sunspot cycle 24 parameters, inferring that it will be only half as active as cycle 23 , reaching its peak in May $2013 \pm 6$ months. The smoothed SSNs for cycle 24 have been rising steadily (albeit slowly) since its onset in December, 2008.

\section{Sunspot Series 1700-2010}

Figure 1 shows a plot of the annual mean SSNs for 17002010, it spans part-Maunder minimum (1645-1715), the Dalton (1800-1830), and the Gleissberg (1889-1902) minima; every fourth cycle is boldly labeled. The Dalton minimum (DM) was preceded by a long cycle 4 (164 months); cycle 23 was 159 months long. The declining phases of cycles 4 and 23 lasted for 123 and 104 months, respectively. Further, one notes that beginning with cycle 10 , there is a pattern where even cycles of the even-odd pairing are less active; it disappears after cycle 21. Also, every third cycle is less active (14, 17, 20, and 23). AJ11 [4] suggest that cycle 24 may lead to a Dalton-like minimum in the 21 st century. We examine the developmental phase of cycle 24 to date and discuss some implications of this outcome, drawing from the historical evidence.

\section{Planetary Indices Ap and aa}

Planetary index Ap was designed by Bartels [5] to measure the geoeffectiveness of the solar corpuscular emission (now solar wind). The index has a linear scale with a range between 0 (quietest day) and 400 (most disturbed day); the maximum value of Ap has never been observed to date, the data go back to 1932. The aa index bears a linear correlation to Ap 


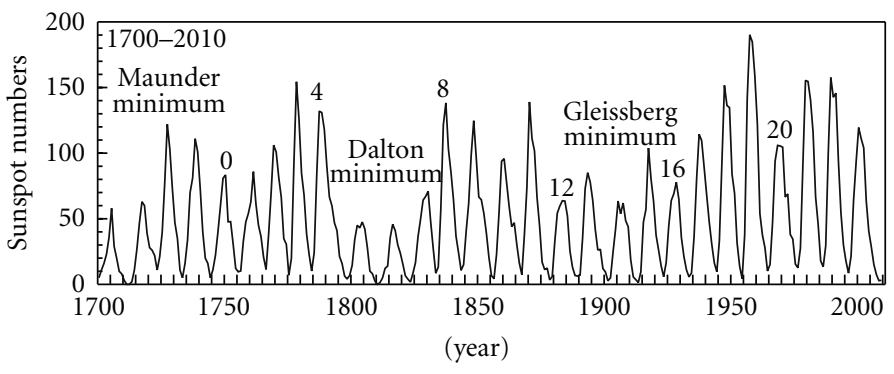

FIGURE 1: SSNs are plotted for 1700-2010; every fourth cycle and the grand minima are labeled.

and covers a longer period to 1868 . AJ11 [4] note that aa index seems to be riding a line of positive slope after the 1901 minimum; no such trend is seen in SSN minima in Figure 1. After 1996 aa/Ap minimum, the trend line changes slope to a steeper negative value; the slope of the trend line before 1901 is also negative. We infer that aa/Ap data may have a period $\sim 200$ years (de Vries/Suess cycle) embedded in them. These indices measure the amplitude of the solar wind electric field [6] at earth orbit, given by the product of the solar wind speed $(V)$ and $B$; in situ measurements of $B$ and $V$ started in October 1963 . The data are readily available at the National Geophysical Data Center website SPIDER of the National Oceanic and Atmospheric Agency at Boulder, CO, USA. AJ11 [4] forecast uses aa/Ap data and the methodology developed by Ahluwalia [7]. This approach led to an accurate forecast for the amplitude of cycle 23 [1].

\section{Grand Minima}

Eddy [8] notes that Maunder minimum (1645-1715), a period of extremely low solar activity in which sunspots became scarce, corresponds to the coldest excursion of the Little Ice Age (1450-1850) in Europe and North America. Reid [9] shows that 11-yr running mean of SSNs bears a close resemblance to the 11-year running average of the measured sea surface temperatures (SSTs) for 1860-1980. Cliver et al. [10] show that there is a correspondence between the trend of the annual mean (aa)min values and SSTs for 18802000. These analyses indicate that there may be a cause-andeffect relationship between the SSNs, (aa)min/Ap, B, and SSTs. More work is needed to clarify the precise relationship between these physical parameters.

Figure 2 shows a plot of the annual mean values of Ap and $B$ for 1963-2009. The positions of the SSN maxima (M) and minima $(\mathrm{m})$ are marked. The vertical dashed lines are drawn through the intervals of the solar polar field reversals. In addition to the Schwabe cycle in the two time series, some additional features are also seen.

(i) The annual mean value of $B$ reached the highest value (9.1 nT) in 1991 (cycle 22); in 2009, it declined to $\sim 2 / 3$ of its value in 1963, the lowest ever measured since the measurements in space began in 1963 . The reader is reminded that data at the Wilcox Solar Observatory (WSO) indicate that solar polar field strength for cycle 23 is significantly lower $(\sim 50 \%)$ than for the previous 3 cycles.

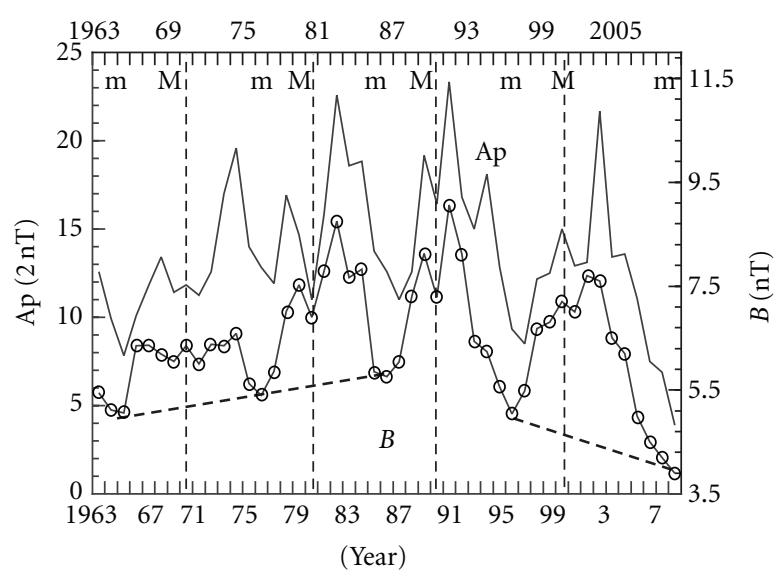

FIgURE 2: Annual mean values of $A p$ and $B$ at earth orbit are plotted for 1963-2009; at the bottom of the graph, the 3-cycle trend is highlighted by dashed lines. See text for details.

(ii) A 3-cycle quasiperiodicity (TCQP) in the limited $B$ data is highlighted in Figure 2 with dashes, with a similar trend in the Ap data. The slope of the trend line changes from positive to a steeper negative value after 1996, as also is the case for aa data (not shown). Ahluwalia [11] discovered the TCQP in an extended shielded ion chamber (IC) data (19371994) and noted its correspondence to TCQP present in the solar and geophysical data over a much longer time interval. He points out that cosmic ray intensity (CRI) minimum in 1939 (cycle 17) is distinctly shallower than CRI minima for cycles 18 and 19 and CRI minimum for cycle 20 is shallower than for cycles 21 and 22. Further, Ahluwalia [12, and references therein] shows that CRI is inversely proportional to $B$. So, TCQP in CRI implies the presence of TCQP in $B$ data string for the period when in situ measurements of $B$ are not available, that is, prior to 1963.

None of the currently available solar dynamo theories are able to account for the TCQP observed in the annual mean $B$ values. Furthermore, the annual mean value $(3.9 \mathrm{nT})$ of $B$ in 2009 is similar to its value in 1912 [4], indicating that we may be returning to the geomagnetic and solar conditions near the early part of the 20th century (Gleissberg minimum), in terms of the interplanetary electromagnetic state at earth orbit. In 2009, CRI reached the highest value [13] observed since the instrumental (IC) measurements began in 1935 . AJ11 [4] infer that if TCQP endures in the latter half of the 


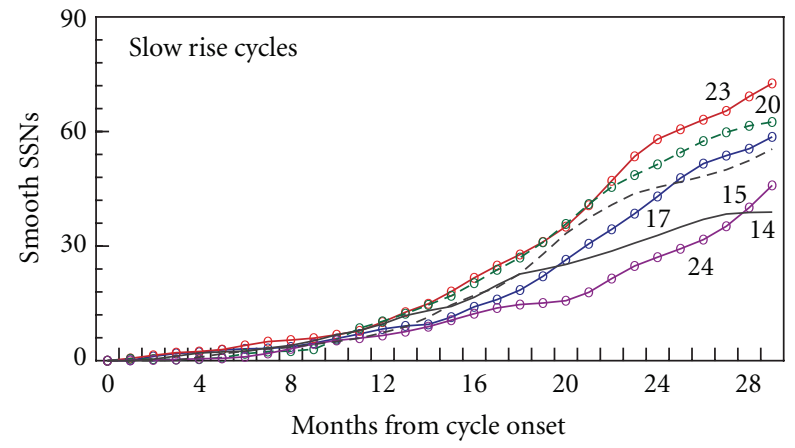

(a)

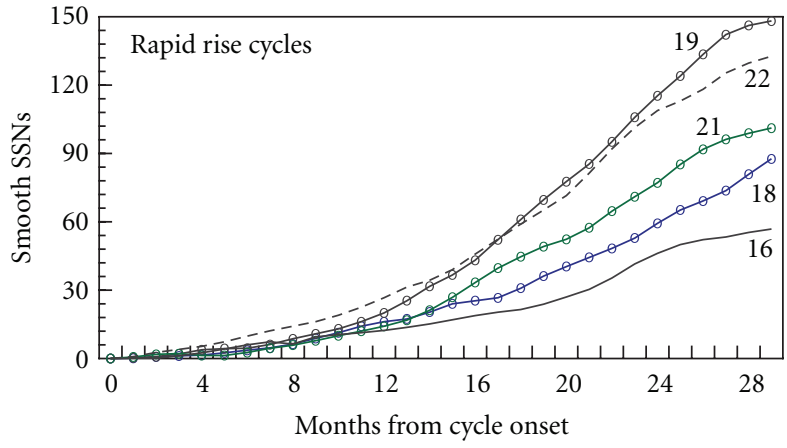

(b)

Figure 3: (a) Timelines for the slow rise cycle of the 20th century are plotted. (b) Timelines for the rapid rise cycles of the 20th century are plotted.

de Vries cycle in the aa/Ap index, we may see the next two sunspot cycles (25 and 26 ) to be successively less active; at this time, we cannot predict whether they will be long or short cycles. It follows that the change in the slope of $B$ for cycle 23 may portend a decreasing trend in SSTs. One is reminded of the prolonged descending phase of cycle 4 leading to DM when earth was cooler. A careful analysis is needed to estimate how the anticipated decrease in SST may compare with an increase in global warming due to an increase in the concentration of the greenhouse gases (such as $\mathrm{CO}_{2}$ ) in the atmosphere and other causes described in the IPCC report.

\section{Timeline Comparisons}

We use smoothed SSNs (to suppress the transients) in describing the profiles of several sunspot cycles of the last century. Figures 3(a) and 3(b) show a comparison between the timelines of cycle 24 activity and ten prior cycles (14-23) for 29 months after the onset. The cycles are normalized at the origin by subtracting the smoothed SSN at the onset from those for the subsequent months. The observed timelines fall into two distinct groups. The cycles 14, 15, 17, 20, and 23 are slow risers like cycle 24; they are depicted in Figure 3(a), and the slow rise cycles have $<100$ smooth SSNs at their peak. The cycles 16, 18, 19, 21, and 22 rise rapidly (compare scales on the left of Figures 3(a) and 3(b)); they have $>100$ smooth SSNs at their peak. They are plotted in Figure 3(b); cycles 18, 19, 21, 22 are very active cycles (see Figure 1 above), 19 being the most active cycle ever observed. Additional points noted are as follows.

For the 29-month period shown in Figure 3(a), the timeline for cycle 23 overlaps with that for cycle 20 since the onset for 21 months (the profiles are barely distinguishable). Early on, cycle 24 follows cycle 17 timeline closely but deviates significantly later on. After 27 months, cycle 24 became more active than cycle 14 . An interesting question is whether cycle 24 may end up being more like cycle 5 that led to the onset of the DM.

Figure 3(b) shows that cycle 22 started out to be more active than cycle 19, but became less so after 18 months. The

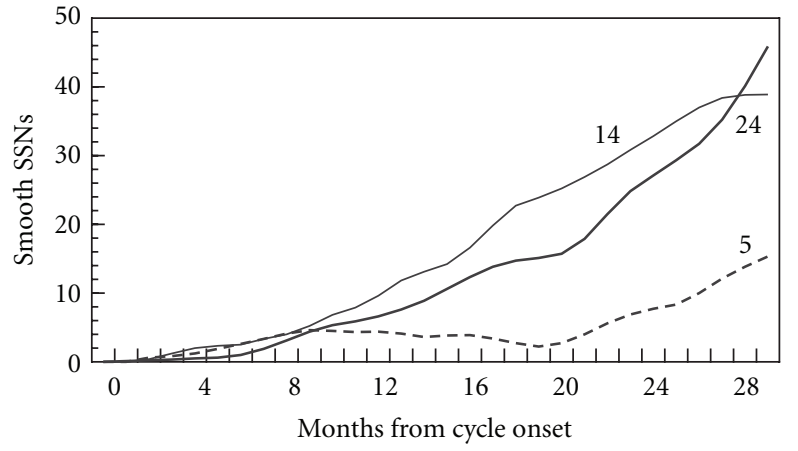

Figure 4: Cycles 5, 14, and 24 compared.

even-odd symmetry in sunspot cycles broke down with cycle 22. Figure 2 shows that the annual mean value of $B$ reached the highest value in cycle 22 and the lowest value in cycle 23. Furthermore, the data at the Wilcox Solar Observatory indicate that the solar polar field strength for cycle 23 is lower $(\sim 50 \%)$ than for the previous three cycles. The peculiar solar behavior during cycles 22-23 is not understood at present.

Figure 4 shows a comparison between the ascending phases of cycles 5, 14, and 24 for 29 months after the onset. Clearly, cycle 5 is the slowest riser; at its peak, the smooth SSN 49.2 may be compared to 56.4 predicted for cycle 24 . Also, cycle 24 seems to follow the timeline of cycle 14, and it led to the onset of the Gleissberg minimum; at its peak, the smooth SSN reached a value of 64.2. At this point in time, it is not clear whether the timeline of cycle 24 will lie betweet those of cycles 5 and 14 . We will follow differences between the three timelines in the future as cycle 24 reaches its peak in 2013.

\section{Global Temperature Change}

Feulner and Rahmstorf [14] use a coupled climate model to explore the future global temperatures. They expect a temperature offset of no more than $-0.30^{\circ} \mathrm{C}$ in 2100 , much smaller than the published estimates for anthropogenic global warming [IPCC, 2001]. 
Akasofu [15] reviews the available data on sea-level changes, glacier retreat, tree-ring observations, ice cores, and changes in CRI from the year 1000 to present. He concludes that the Little Ice Age (LIA) occurred in Asia as well. He infers that the global recovery from the LIA has proceeded continuously (in about a linear manner) from 1800-1850 to the present @ $0.5^{\circ} \mathrm{C} /$ century. Akasofu predicts that the temperature increase in 2100 will be $0.5^{\circ} \mathrm{C} \pm 0.2^{\circ} \mathrm{C}$ in contrast to $4^{\circ} \mathrm{C} \pm 2^{\circ} \mathrm{C}$ predicted by the IPCC. An interesting possibility presents itself. If Akasofu and Fuelner and Rahmstorf are both right, we may end up with little net long-term temperature change in the year 2100.

Lean et al. [16] show that the total solar irradiance (TSI) decreased significantly during the DM; the decrease was even more remarkable for the Maunder minimum (MM) for an extended time period (see Figure 2). Since these estimates are all proxy based, the relevant science is far from settled yet. For example, Schrijver et al. [17] suggest that TSI during that period may not have been as low as previously thought. They analyze direct measurements of solar magnetic activity during the recent 2008-2009 period of low sunspot activity, which they argue is similar to the activity level during the MM. They find that even when there are no sunspots, the sun has a baseline level of magnetic activity. This baseline has not been taken into account in previous estimates of TSI during the MM, which are based solely on SSNs. The authors suggest that earlier estimates of the TSI during the MM are too low. They argue that MM probably had levels of magnetic activity and TSI similar to the 2008-2009 values. So, factors other than low TSI resulting from low sunspot activity must have contributed to the LIA, inferring that drivers other than TSI dominate the long-term climate change on earth.

Ahluwalia [18] argues that if solar wind existed during the $\mathrm{MM}$, the value of $B$ must have been $\geq 3.3 \mathrm{nT}$. In our opinion, the role of $B$ in contributing to the global climate change is neither fully appreciated nor understood. IPCC undervalues the forcing arising from changes in solar activity and $B$ which may turn out to be much more important than the forcing from $\mathrm{CO}_{2}$.

There is a great interest in understanding the relationship between cosmic ray caused ionization in the atmosphere and the cloud cover [19-22, and references therein]. The underlying hypothesis (unverified experimentally) is that cosmic rays, modulated by solar activity, influence cloud formation and therefore temperature. So, the natural versus anthropogenic causes of global warming debate is likely to continue for a while, yet we still do not know as much as we think we know. The challenges posed by the climate change can only be addressed by interdisciplinary efforts, using highquality data. Perhaps we stand at the crossroads.

Our heuristic analysis (which is not based on any model) cannot rule out the premise that cycle 24 may herald the onset of a DM-like minimum in the present century [4].

6.1. Probable Socioeconomic and Political Scenarios. It is interesting to note that the interval of the DM coincided with a significant rise in inflation in England, for example, the price of wheat rose by a factor $\sim 2$ [23]. Considering that human population is projected to reach 10 billion in the 21 st century, the higher food prices (if they occur) may cause social and political upheaval in the overpopulated countries of Asia and Africa; people living in the towns of sub-Saharan Africa spend a bigger share of their income on food than do urban residents almost anywhere else in the world. Laborers use up over half their wage just to eat. So, they will spend less on school fees, sanitation, and health.

The food, fuel, and climate crises are by themselves serious issues, but taken together, their impact could be disastrous for the world economy and intranational and international conflicts, threatening the security of individuals and nations. Optimistically speaking, we may hope that advances in sciences and the agricultural technology in the 21st century may meet the challenge of food shortage and mitigate the adverse circumstances forecast by Thomas Robert Malthus FRS (1766-1834) nearly two centuries ago. In this context, we note further that there were remarkable strides in the scientific enterprise during DM, for example, advances in electromagnetism and thermodynamics. The University of Berlin was founded in 1810; it led to a new model for science teaching, later copied worldwide.

\section{Summary}

We have examined the progress of SSN cycle 24 activity since its onset in December, 2008 and compared it with the timelines of the last ten solar cycles (14-23) of the 20th century. We find that cycle 24 is the slowest riser. According to Waldmeier [24], the rise time of a cycle is determined by a single parameter, namely, the SSN at its peak; he showed that active cycles have a steeper rise and moderate cycles rise more slowly. Ahluwalia [7] certified the validity of the Waldmeier effect for seven cycles (17 to 23) of the 20th century, showing that moderate cycles attain their maximum an average of eight months later and have a tendency to linger near the maximum. The physical cause of the Waldmeier effect and its relationship to the workings of the solar dynamo is not known. Nevertheless, the observed trend to date of cycle 24 timeline strongly suggests that AJ11 [4] forecast of very low activity for cycle 24 is quite reasonable and likely to come true. We have discussed the implications of this outcome on the future earth climate change and the socioeconomic and political scenarios drawing from the past history.

\section{Acknowledgments}

The authors are grateful to the providers of solar, interplanetary, and geomagnetic data used in this study. One referee made useful suggestions. This research is supported by NASA EPSCoR Award NNX09AP76A-Q01378.

\section{References}

[1] K. Petrovay, 7, 1-63, 2010, http://arxiv.org/PS_cache/arxiv/pdf/ 1012/1012.5513v2.pdf.

[2] A. I. Ohl, "Wolf's number prediction for the maximum of the cycle 20," Solnechnye Dannye (solar data, in Russian) Bulletin, vol. 12, pp. 84-85, 1966. 
[3] A. I. Ohl, "A preliminary forecast of some parameters of 21 solar cycle activity," Solnechnye Dannye Bulletin, vol. 9, pp. 7375, 1976.

[4] H. S. Ahluwalia and J. Jackiewicz, "Sunspot cycle 23 descent to an unusual minimum and forecasts for cycle 24 activity," Advances in Space Research. In press.

[5] J. Bartels, Collection of Geomagnetic Planetary Index Kp and Derived Daily Indices Ap and Cp for the Years 1932-1961, North Holland, New York, NY, USA, 1962.

[6] H. S. Ahluwalia, "Ap time variations and interplanetary magnetic field intensity," Journal of Geophysical Research A, vol. 105, no. 12, pp. 27481-27487, 2000.

[7] H. S. Ahluwalia, "Meandering path to solar activity forecast for cycle 23," in Proceedings of the 10th International Solar Wind Conference, M. Velli, R. Bruno, and F. Malra, Eds., vol. CP679, pp. 176-179, The American Institute of Physics, 2003.

[8] J. A. Eddy, "The maunder minimum," Science, vol. 192, no. 4245, pp. 1189-1202, 1976.

[9] G. C. Reid, "Influence of solar variability on global sea surface temperatures," Nature, vol. 329, pp. 142-143, 1987.

[10] E. W. Cliver, V. Boriakoff, and J. Feynman, "Solar variability and climate change: geomagnetic aa index and global surface temperature," Geophysical Research Letters, vol. 25, no. 7, pp. 1035-1038, 1998.

[11] H. S. Ahluwalia, "Three activity cycle periodicity in galactic cosmic rays," in Proceedings of the 25th International Cosmic Ray Conference, vol. 2, pp. 109-112, Durban, South Africa, 1997.

[12] H. S. Ahluwalia, "Cycle 20 solar wind modulation of galactic cosmic rays: understanding the challenge," Journal of Geophysical Research A, vol. 110, no. 10, Article ID A10106, 2005.

[13] H. S. Ahluwalia and R. C. Ygbuhay, "The onset of sunspot cycle 24 and galactic cosmic ray modulation," Advances in Space Research, vol. 48, no. 1, pp. 61-64, 2011.

[14] G. Feulner and S. Rahmstorf, "On the effect of a new grand minimum of solar activity on the future climate on Earth," Geophysical Research Letters, vol. 37, Article ID L05707, 2010.

[15] S. Akasofu, "On the recovery from the little ice age," Natural Science, vol. 2, pp. 1211-1224, 2010.

[16] J. Lean, J. Beer, and R. Bradley, "Reconstruction of solar irradiance since 1610: implications for climate change," Geophysical Research Letters, vol. 22, no. 23, pp. 3195-3198, 1995.

[17] C. J. Schrijver, W. C. Livingston, T. N. Woods, and R. A. Mewaldt, "The minimal solar activity in 2008-2009 and its implications for long-term climate modeling," Geophysical Research Letters, vol. 38, Article ID L06701, 6 pages, 2011.

[18] H. S. Ahluwalia, "Timelines of cosmic ray intensity, Ap, IMF, and sunspot numbers since 1937," Journal of Geophysical Research, vol. 116, Article ID A12106, 9 pages, 2011.

[19] E. P. Ney, "Cosmic radiation and weather," Nature, vol. 183, pp. 451-452, 1959.

[20] J. T. A. Ely, J. J. Lord, and F. D. Lind, "Annual modulation of galactic cosmic rays circa $1 \mathrm{GV}$ and relevance to tropospheric processes," in Proceedings of the 23rd International Cosmic Ray Conference, vol. 3, pp. 586-589, Calgary, Canada, 1993.

[21] H. Svensmark and E. Friis-Christensen, "Variation of cosmic ray flux and global cloud coverage-a missing link in solarclimate relationships," Journal of Atmospheric and SolarTerrestrial Physics, vol. 59, no. 11, pp. 1225-1232, 1997.

[22] H. Svensmark, "Influence of cosmic rays on Earth's climate," Physical Review Letters, vol. 81, no. 22, pp. 5027-5030, 1998.

[23] D. H. Menzel, Our Sun, Harvard University Press, Cambridge, Mass, USA, 1959.
[24] M. Waldmeier, Ergebnisse und Probleme der Sonnenforschung, Leipzig, Germany, 2nd edition, 1955. 

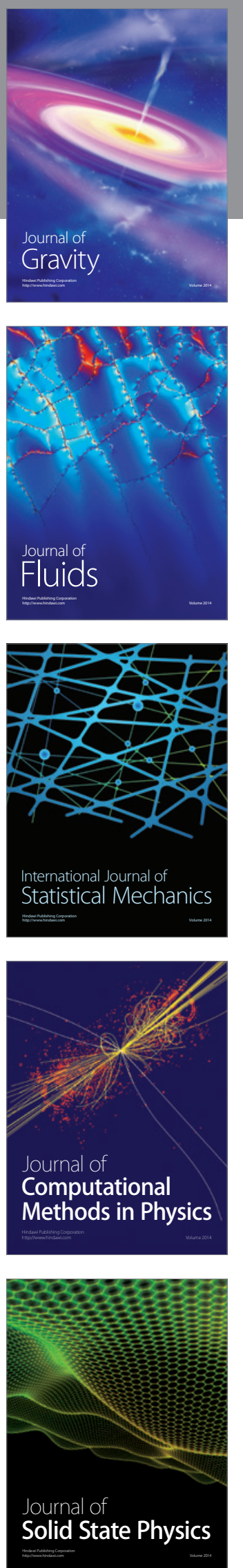

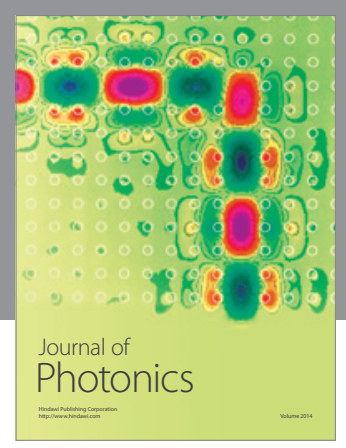

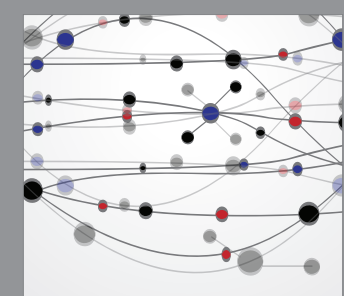

The Scientific World Journal
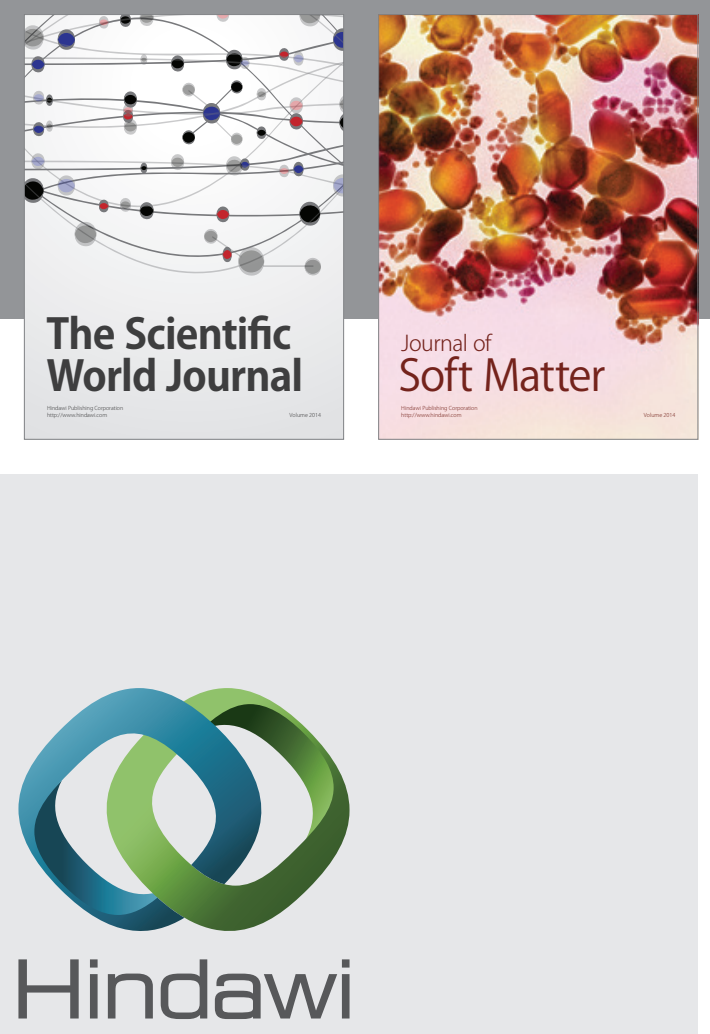

Submit your manuscripts at

http://www.hindawi.com
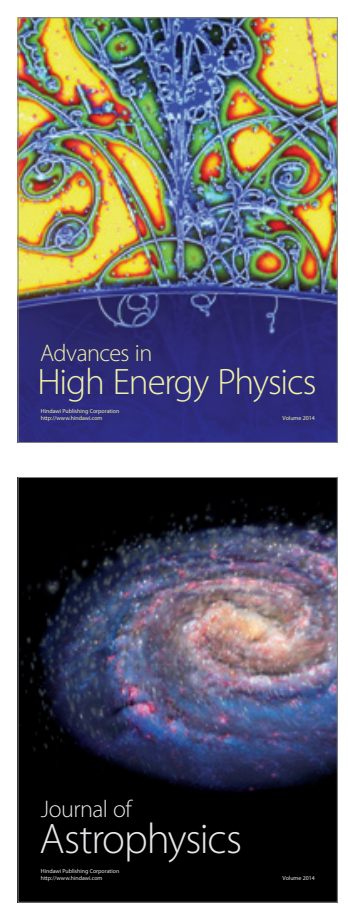
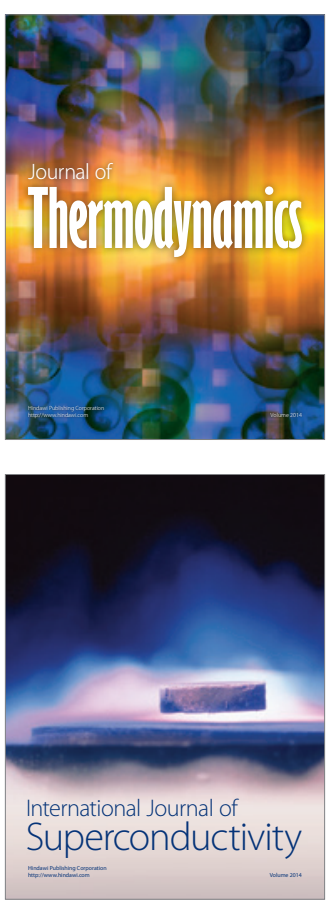
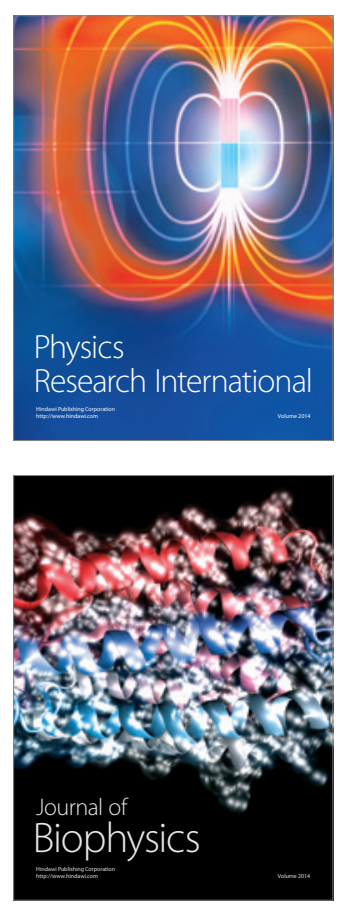
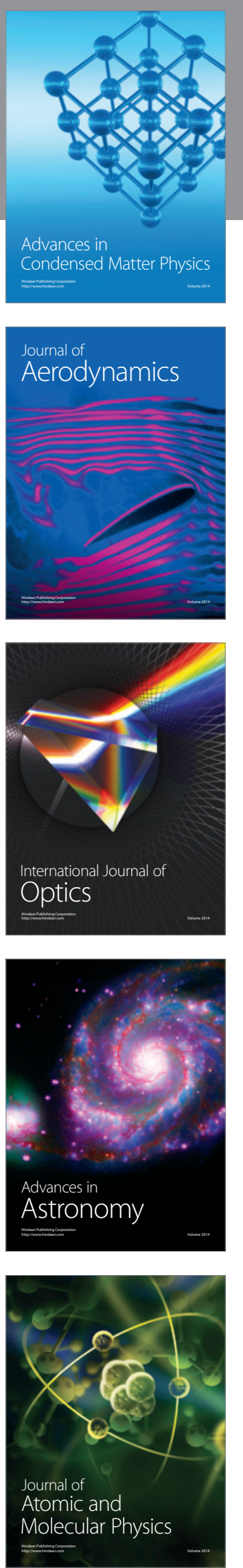